\title{
REVIEW ON BONE DISEASE (OSTEOPOROSIS) IN DIABETES MELLITUS
}

\author{
By \\ AHMED HASSAN FAWZI EL-TAWDY ${ }^{1}$, EISSA ABDEL HAMED IBRAHIM ${ }^{1}$, \\ EMAN MAHMOUD ALY AL SAKHAWY ${ }^{1}$ and TOSSON A. MORSY ${ }^{2}$ \\ Military Medical Academy, Cairo $11291^{1}$, and Faculty of Medicine, \\ Ain Shams University, Cairo $11566^{2}$, Egypt
}

Abstract

Osteoporosis is the most common bone disease known, affecting an estimated 200 million people worldwide. Approximately $30 \%$ of all postmenopausal women are affected and up to $40 \%$ developed a fragility fracture within their lifetime. Over time, a number of risk factors have been associated with osteoporosis and are useful when used in screening tools and treatment algorithms. Diabetes, although identified more than half a century ago as being associated with bone frailty, has come to the forefront only within last decade as an important osteoporosis risk factor. While both type 1 (DM1) and type 2 (DM2) diabetes increased fracture risk, the link is far more profound with DM1. DM1 accounts for approximately 5\% of all cases of diabetes in the USA and is associated with a 6.4-6.9-fold increase in the relative risk of hip fracture compared to individuals without diabetes. As life expectancy continues to increase for those living with DM1, an increase in number of fractures occurring in this population is expected in the future. For understanding of bone fragility in DM1 calls for an update of diabetes guidelines, better screening tools, and more research into the use of therapeutic strategies in these patients.

Key words: Osteoporosis, Diabetes mellitus, type 1 and type 2, Man, Woman.

\section{Introduction}

The link between type 1 diabetes mellitus (DM1) and osteoporosis recently identified gained attention. While a number of cellular mechanisms were postulated to mediate this association, it is now established that defects in osteoblast differentiation and activity are the main culprits underlying bone fragility in DM1. Other factors include an accumulation of advanced gyration end products (AGEs) and diabetes complications development (as neuropathy \& hypoglycemia) causing more decline in bone mineral density (BMD), worsening geometric properties within bone, and the increased fall risk.

Consequently, DM1 patients have a 6.9fold increased incidence of the hip fracture compared to controls. Despite this increased fracture risk, bone fragility remains the under-appreciated complication of DM1 and not addressed in most diabetes guidelines. Also, there was a lack in knowledge regarding the efficacy of therapeutic strategies to treat osteoporosis in this patient population (Khan and Fraser, 2015). Although the metabolic abnormalities of diabetes potentially affect bone metabolism, structure, and mineral density, it is not clear if these changes are responsible for the increase in fracture risk noted in individuals with type 1 and type 2 diabetes, besides changes in bone metabolism, there are several other factors that may be important: The degree of bone loss may differ between type 1 and 2 diabetes which were associated with an increase in bone mineral density (Tuominen et al, 1999).

\section{Review and General Discussion}

Bone metabolism may be affected by late complications of diabetes (e.g., renal failure) the risk of fracture may be increased by falling secondary to visual impairment, cerebrovascular disease, or the neuropathy The localized bone loss related to diabetic neuropathy may increase the risk of fracture at the foot and ankle. The specific treatments may influence the fracture rates.

A variety of musculoskeletal conditions have been associated with diabetes mellitus (show figure). Specific arthropathies of the hand and shoulder are discussed in this review. The relationship of diabetes with osteoarthritis is not so clear and the data that 
bear on the association, or lack thereof, are also briefly mentioned.

1- Hand abnormalities are common in the diabetic patients, reflecting the pathologic changes in the microvasculature, connective tissue, and peripheral nerves. A total of 25/ 50 patients with hand syndromes were disabled to such an extent that surgery was recommended (Gamstedt et al, 1993). In Type $1 \& 2$ diabetes, the prevalence of hand or shoulder disorders was higher in diabetic patients than in controls and correlated with duration but not diabetes' type (Cagliero et al, 2002). Redmond et al. (2012) observed the natural history of hand function during a two-year period in participants with hand syndromes associated with diabetes and to determine factors related to changing the function. They found that light touch perception reduced in longstanding diabetic hand syndromes. Tactile abnormalities that were detected by clinical examination progressed during a two year period and were related to metabolic control and musculoskeletal diagnosis

2- Carpal tunnel syndrome (CTS) is a painful disorder caused by median nerve compression between the carpal ligament and other structures within the carpal tunnel, and was reported in up to $20 \%$ of diabetic patients, and up to $75 \%$ in those with limited joint mobility (Chaudhuri et al, 1989). Also, CTS may be commonest in those with prediabetes (Gulliford et al, 2006). Median nerve entrapment in diabetes is presumably due to the connective tissue changes that lead to limited joint mobility and associated with diabetes duration but without metabolic control, nephropathy, or retinopathy (Graham et al, 2004).

3- Dupuytren's contracture is characterized by fibrosis in and around the palmar fascia, with nodule formation and contracture of palmar fascia leading to flexion contractures of the digits. Many associations have been reported, including racial and genetic factors, chronic liver disease, and diabetes (Sibbitt, 1989). Based on diagnosis criteria,
Dupuytren's contractures were reported in 16 to $42 \%$ of the diabetic out-patients, prevalence increased with age and duration (Noble et al, 1984). Bhavsar et al. (2009) reported that athero-sclerosis, a response to injury, might be thought of as scarring in the artery wall. TGF-beta and associated signaling molecules were implicated in the pathophysiology of keloid scarring, Dupuytren's Contracture and atherosclerotic plaques in independent studies. They concluded that keloid scarring is a clinical clue to excess atherosclerosis not explained by traditional risk factors. Such patients might benefit from therapy directed at targets related to signaling molecules common to process of keloid scarring and athero-sclerosis, and recommended that unexplored possibilities for prevention and treatment of atherosclerosis

4- Flexor tenosynovitis: Tenosynovitis of the flexor tendon of the finger (trigger finger) in diabetes is characterized by palpable nodule formation and thickening localized to the flexor tendon or sheath, and locking phenomena (5\% aged 3 to 38 years type 1 diabetes and $20 \%$ aged 19 to 62years patients with type 1 or type 2 diabetes). The ring, middle fingers, and thumb mostly affected and sometimes bilateral (Yosipovitch et al, 1990). The prevalence of trigger finger is related to the duration of diabetes but not metabolic control. The collagen abnormalities induced by diabetes mellitus are thought to be responsible. The local glucocorticoid injection may be beneficial but some patients require repeated surgery (Kapoor and Sibbitt, 1989).

5- Diabetic sclerodactyly: Sclerodactyly is characterized by thickening and waxiness of the skin most marked on the dorsa of the fingers, with limited joint mobility, although it can occur without joint involvement. The skin changes resemble those in scleroderma, but the Raynaud phenomena, ulceration, calcinosis and tapering are absent and autoantibodies are negative; pseudoscleroderma. The skin changes always correlates with the duration of diabetes, although skin changes 
were described in children with recent onset diabetes (Jelinek, 1993). Gruson and Franks (2005) reported 40-year-old man with hardening of skin of his hands and upper back, which had slowly worsened with time. His medical history included the insulin-dependent diabetes mellitus since childhood. Histopathologic features of a biopsy sample from the skin of his back showed a thick reticular dermis with collagen bundles in a haphazard array, which were separated by increased deposits of connective-tissue mucin. The scleredema and diabetic sclerodactyly are well recognized skin findings that may occur in patients with diabetes mellitus. It is important to differentiate this condition from the scleroderma. Treatment is difficult, and therefore many modalities have been used.

6- Reflex sympathetic dystrophy: Reflex sympathetic dystrophy (or complex regional pain syndrome) characterized by severe pain or burning sensation, commonest in hand or foot, with associated swelling, trophic skin changes, and signs or symptoms of vasomotor instability. When the hand is affected, there may be associated pain or limitation of the ipsilateral relex shoulder (shoulder-hand syndrome). Reflex sympathetic dystrophy may be bilateral, and the bone films characteristically show patchy osteoporosis, but less common with diabetes (Kozin, 1998).

7- Shoulder pain: Two main types of the shoulder problem, adhesive capsulitis (frozen shoulder) and calcific periarthritis (calcific tendinitis), were described in diabetic patients. Adhesive capsulitis was reported in 19 to $29 \%$ of diabetics compared to $5 \%$ of controls (Pal et al, 1986). Longer duration of diabetes, increased age, limited joint mobility, and Dupuytren's contractures were also associated with an increased risk of painful capsulitis; retinopathy was also more frequent in those with capsulitis, but neither neuropathy nor proteinuria (Balci et al, 1999). Soft tissue calcification with shoulder periarthritis also appeared to occur three times more frequently in diabetics than controls although only one-third was symptomatic
(Mavrikakis et al, 1999). Adhesive capsulitis is a common cause of shoulder pain; in 60 diabetic patients with shoulder pain, $58 \%$ had adhesive capsulitis and $28 \%$ had tendinitis (Moren-Hybbinette et al, 1987). A link between adhesive capsulitis and limited joint mobility was postulated, but limited joint mobility affected shoulder joint might not be a true capsulitis (Fisher et al, 1986).

8- Osteoarthritis: Obesity is a risk factor for both osteoarthritis of knee and NIDDM (Hochberg, 1991), but not clear if diabetes mellitus is a risk factor for the osteoarthritis, independent of obesity (Horn et al, 1992),

9- Limited joint mobility: Limited joint mobility is common in patients with diabetes mellitus, characterized by limitation of joint movement mainly in the hands' small joints (Jelinek, 1993) with the thickening and waxiness of the skin commonest particularly on the fingers' dorsal surface, but changes may occur in the absence of limited joint mobility (Seibold, 1982).

10- Neuropathic arthropathy: A variety of musculoskeletal conditions are associated with diabetes mellitus, including specific arthropathies of the hand and shoulder, spontaneous infarction of skeletal muscle, and limited joint mobility. The loss of sensation to a joint may result in a chronic, progressive, and destructive arthropathy. The prototype of this disorder was described by Charcot in relation to tabes-dorsalis. Similar the changes are seen with other neurologic disorders, such as syringomyelia and diabetic neuropathy. Diabetes is now commonest cause of neuropathic (Char-cot) arthropathy in the Western world. Diabetic neuropathic arthropathy is also called diabetic osteoarthropathy (Baumhauer et al, 2006).

11- Bone Disease: Although metabolic abnormalities of diabetes potentially affect bone metabolism, structure, and mineral density, it was not clear if these changes are responsible for the increase in fracture risk noted in individuals with type 1 and type 2 diabetes. The degree of bone loss may differ between type 1 and type 2 diabetes, which 
may be associated with an increase in bone mineral density, may be affected by the late complications of diabetes e.g., renal failure (Tuominen et al, 1999). Length-dependent neuropathy is the commonest and costly complication of diabetes and frequently causes injury primarily to the small-diameter cutaneous nociceptive fibers. Not only the persistent hyperglycemia but also the metabolic, endocrine, and inflammatory effects of obesity and dyslipidemia play an important role in diabetic neuropathy development (El-Tawdy et al, 2015). Rational therapies aimed at direct control of glucose or its increased entry into the polyol pathway, oxidative or nitrosative stress, advanced glycation end product formation or signaling, microvascular ischemia, or adipocyte-derived toxicity all failed in human trials of diabetic neuropathy. Aerobic exercise produces salutary effects in many of these pathogenic pathways simultaneously and, in both animal models and human trials, has been shown to improve symptoms of neuropathy and promote re-growth of cutaneous smalldiameter fibers (Singleton et al, 2015).

12- Diabetic muscle infarction: One of the commonest causes of skeletal muscle infarction is diabetic muscle infarction (DMI), a rare complication associated with poorly controlled diabetes. Mimata et al. (2015) reported an atypical case of DMI localized in the tibialis anterior (TA) and extensor hallucis longus (EHL) muscles of a 64-year-old man with type 2 diabetes mellitus presented with a 6-month history of a palpable mass in his lower left leg. Magnetic resonance imaging (MRI) revealed that the mass exhibited heterogeneous signals on T1- \& T2-weighted images and slight heterogeneous enhancement within the muscles on fat suppressed T1-weighted images. As histopathological analysis showed mostly necrotic muscle tissues but no neoplastic cells, the affected muscles were resected. A typical symptom of DMI is severe abrupt-onset pain in the region of the affected muscles, but the patient did not complain of pain. Thus, diag- nosis and treatment for DMI were delayed, and widespread irreversible muscle necrosis developed. MRI findings of DMI can be similar to that of a malignant soft-tissue tumor. They concluded that to consider the malignant soft-tissue tumor as one of the differential diagnoses of DMI is a must.

Bone metabolism in diabetes: Studies in bone histo-morphometry in type 1 diabetes have generally, but not always (McNair, 1988) shown a low turnover of bone with reductions in bone formation and, to a lesser degree, bone resorption. Decrease in bone formation is manifested by reduced serum concentrations of osteocalcin, a marker of osteoblastic activity. In comparison, resorption markers (such as serum tartrate resistant acid phosphatase and urinary hydroxyproline) are increased in some patients (Olmos et al, 1994), perhaps related to alterations in renal function (Selby et al, 1995). Similar changes were noted in diabetic patients who progress to end-stage renal disease. The compared to normal subjects, patients with diabetes are more likely to have adynamic bone disease; including that associated with aluminum deposition (Pei et al, 1993), while hyperparathyroid bone disease occurred in less than $10 \%$ of cases (Jara et al, 1995).

In uremic rats that lack of insulin might diminished bone turnover. But, insulin deficiency might be earlier important, since decreased bone formation can be demonstrated in type 1 diabetics before the clinical onset of renal disease (Krakauer et al, 1995).

Low bone turnover, with a reduction in unmineralized bone matrix, and increased collagen glycosylation may contribute to increased fragility of the diabetic bone (Hernigou et al, 2015) reported that in diabetic patients with ankle non-unions, treatment with BM-MSCs from bone marrow concentrate may be preferable in view of the high risks of major complications after open surgery and iliac bone grafting, and improved healing rates compared with standard iliac bone autograft treatment. Another factor that can affect bone metabolism in diabetes is 
hypercalciuria. The mechanism by which this occurs is not clear but it can be ameliorated with insulin. Both loss of bone mineral and functional hypoparathyroidism may play a contributory role. Dominguez et al. (2004) stated that diabetes may affect bone tissue by different mechanisms including obesity, hyperinsulinemia, advanced deposit of glycosilation end products in collagen fibre, reduced circulating levels of IGF-1, hypercalciuria, renal function impairment, microangiopathy and chronic inflammation..

Bone density in type 1 diabetes: Children and adolescents with type 1 diabetes have reduced bone mineral density (BMD) at the forearm which appeared constant with time (Lettgen et al, 1995),. It is less clear whether this deficit is present at the lumbar spine. Variable findings have been reported ranging from reductions in trabecular but not cortical BMD [18] to no deficit reported when BMD is corrected for weight. It was hypothesized that any defect in BMD is due to a failure to acquire endosteal bone during growth (Compston et al, 1995).

In the adults with type 1 diabetes, lumbar BMD is usually normal Mastrandrea et al, 2008) but femoral BMD is reduced (Kayath et al, 1995). Usually there was no relationship between BMD and diabetes duration or glycemic control, but not uniformly found (Rishaug et al, 1995). Premenopausal women showed increased lumbar BMD and normal femoral BMD. In another study, lumbar BMD was low; a high proportion of patients with retinopathy and neuropathy, suggested that micro-vascular disease can influence BMD and alink between neuropathy and low femoral neck BMD (Forst et al, 1995).

Bone density in type 2 diabetes: Majority of studies in type 2 diabetes have found that BMD is normal or increased at the lumbar spine (Van Daele et al, 1996), femoral neck and mid and distal radius (Barrett-Connor and Holbrook, 1992). As expected, BMD correlated with body mass index, but in the studies that corrected for the body mass index, increased BMD was independent of the obesity (Wakasugi et al, 1993).

A study of older well-functioning type 2 diabetics showed higher hip, total body, and volumetric spine BMD in black and white men and women compared with their appropriate controls, although a lower spine bone volume was found in diabetes patients which may possibly decrease bone strength (Strotmeyer et al, 2004).

A longitudinal 12-year study of 109 patients with type 1 and type 2 diabetes reported two major findings: Radial BMD was lower than in normal subjects in both groups at baseline. The rate of decline in radial BMD over time was similar to that in normal subjects in type 1 diabetes and less than that in normal subjects in type 2 diabetes such that the original deficit was reversed. So, impaired osteoblast recruitment or function was responsible for the original reduction in BMD and that low bone turnover diminished age-related bone loss in older patients with type 2 diabetes. One limitation of these findings is that BMD could only be measured in survivors, which comprised just over one-half of the original study population. One group of patients with type 2 diabetes who appear to be at risk for an increased rate of bone loss was women treated with thiazolidinediones.

Safety with thiazolidinediones: 1- All of the thiazolidinediones cause weight gain. It was dose-dependent and time-dependent and can be substantial (Akazawa et al, 2000).

The weight gain is caused in part by fluid retention. A small study $(n=8)$ found that 12 weeks of therapy with pioglitazone $45 \mathrm{mg}$ resulted in 2.4 liters of water accumulation, which accounted for $75 \%$ of $3.1 \mathrm{~kg}$ weight gain by Fluid retention/heart failure (Basu et al, 2006). Weight gain may also result from the proliferation of new adipocytes. The upregulation of genes that facilitate adipocyte lipid storage in vivo was described in one study (Bogacka et al, 2004). There is a suggestion from one cohort of patients taking pioglitazone continuously for over 30 months with average weight gain increases stea- 
dily up to 30 months but may plateau (at around $5.3 \mathrm{~kg}$, or 12 pounds) by 36 months (King et al, 2003). In a trial of 39 insulinresistant, non-diabetic subjects with the central obesity randomly assigned to diet and exercise, or pioglitazone ( $30 \mathrm{mg} /$ day) for 20 weeks, insulin sensitivity improved in both groups. Patients on diet and exercise group lost a mean of $11.8 \mathrm{~kg}$ while the pioglitazone group gained $2.7 \mathrm{~kg}$. Both groups showed a decrease in the waist-to-hip ratio but in the pioglitazone group this was due to a preferential increase in lower body fat deposition. Only diet and exercise were associated with a decrease in intraabdominal fat (Shadid and Jensen, 2003).

Among 1159 type 2 diabetic patients taking metformin, $5 \mathrm{mg}$ of dual PPAR agonist muraglitazar caused an increase in weight at 50 weeks of $2.5 \mathrm{~kg}$, compared with an increase of $1.5 \mathrm{~kg}$ for patients treated with $30 \mathrm{mg}$ of pioglitazone (Kendall et al, 2006).

Peripheral edema occurs in 4 to $6 \%$ of patients treated with the thiazolidinediones (compared to 1 to $2 \%$ with placebo) and in a higher percentage of patients with a history of heart failure (HF). The thiazolidinediones act by binding to and by activating PPARgamma is abundant in the collecting tubules, and the fluid retention with thiazolidinediones resulted from the PPAR-gamma stimulation of sodium reabsorption by sodium channels (called epithelial sodium channel) in luminal membrane of the collecting tubule cells. This effect is mediated by increased expression of gamma subunit of sodium channel gene mRNA (Guan et al, 2005).

Sodium retention by aldosterone is also mediated by this channel. However, the aldosterone excess is not associated with edema, due to the phenomenon of aldosterone escape in which sodium reabsorption is reduced at other sites in the nephron to minimize fluid retention. Since the thiazolidinediones act at the same site as aldosterone, observations with aldosterone excess suggest that edema or heart failure associated with thiazolidinediones requires additional factors, such as underlying heart disease. Due to HF risk, the American Heart Association (AHA) and the American Diabetes Association (ADA) published a con-sensus statement in 2003 on thiazolidinedione use, fluid retention, and HF (Nesto et al, 2003).

Skeletal: There is an increasing body of evidence suggested that thiazolidinediones decrease bone density and increase fracture risk especially in women (Home et al, 2009). In animal models, treatment with the rosiglitazone resulted in bone loss by the suppression of osteoblast differentiation and formation (Ali et al, 2005). The bone loss was associated with an increase in marrow adipocytes. In vivo studies suggest that this finding is mediated by rosiglitazone induced activation of PPAR-gamma. PPAR-gamma2 isoform is an important regulator of adipocyte differentiation. Activation of PPARgamma2 results in diversion of bone marrow stromal cells from the osteoblast lineage into the adipocyte lineage, which subsequently leads to a decrease in bone formation rates and increase in adipogenesis (Rzonca et al, 2004). This process may be regulated by IGF system, as rosiglitazone induced activation of PPAR-gamma was demonstrated to down regulate components of IGF system in vivo \&in vitro, and IGF-1 is an important regulator of osteoblast proliferation and differentiation (Lecka-Czernik et al, 2007).

Fracture Risk: Although some studies have not found a relationship, the majority have demonstrated an increased risk of fracture in patients with diabetes (Yamamoto et al, 2009): In the Women's Health Initiative Observational Study, a prospective cohort study of 93,676 postmenopausal women, women with type 2 diabetes $(n=5285)$ had an increased risk of fracture after seven years of follow up compared with women without diabetes (adjusted relative risk [RR] 1.20, 95\% CI 1.11-1.30). A systematic review and a meta-analysis of diabetes \& fracture showed similar increases in risk of hip fracture in patients with either type 1 diabetes; RRs 6.3 $\& 6.9$, respectively or type 2 diabetes; RRs 
$1.7 \& 1.4$, respectively (Janghorbani et al, 2007). Fracture risk was higher and BMD lower in those with diabetic complications.

An association between risk of fractures, particularly proximal humerus fractures and diabetic retinopathy, advanced cortical cataract, longer diabetes duration and insulin treatment has been described. Association between retinopathy and the fracture risk remained significant after adjusting for the visual acuity and it was suggested that retinopathy may be acting as a proxy for severe diabetic microvascular disease. An increased risk of falling in older diabetic women has been demonstrated (Leslie et al, 2007). An increased incidence of foot fractures, predominantly metatarsal was also $\backslash$ descry-bed in diabetic athletes in a retrospective study. Pierre-Jerome et al. (2012) stated that despite not bearing weight, cuboid bone is more vulnerable to marrow edema and fractures in diabetic patients compared to nondiabetic patients. Age seems to be an influential factor. Fractures were more common in men and were associated with a long duration of disease. In addition, diabetic neuropathy has been associated with osteopenia in both hands and feet, metatarsal fractures, and a subsequent risk of osteopathy (Vestergaard et al, 2005). Possibility of fracture risk was associated with disease duration and presence of long-term complications showed by a retrospective population-based cohort study of 82,000 adults with diabetes and over 236,000 matched non-diabetic controls (Loder, 1988). The risk of osteoporotic (RR $1.15,95 \%$ CI 1.09-1.22) and hip (RR 1.40, 95\% CI 1.28-1.53) fractures was increased in adults with long-term diabetes, whereas it was decreased in newly diagnosed diabetes (RRs 0.91, 95\% CI 0.86-0.95 and 0.83, 95\% CI 0.75-0.92 for osteoporotic \& hip fractures, respectively). Fracture risk may also be related to therapy. A diabetes outcome progression trial reported a higher rate of fractures in newly diagnosed women with diabetes randomly assigned to receive four years of rosiglitazone, as opposed to the me- tformin or glyburide. Taken all these data into consideration suggested that BMD was lower in patients with type 1 diabetes and normal or increased in patients with type 2 . But, fracture risk appears increased in both groups, possibly related to factors in addition to BMD, as diabetes duration and complications, treatment, and risk of falling.

Fracture healing: Prolonged fracture healing was described in both type 1 and type 2 diabetes. This occurred in patients with displaced fractures and those treated by open reduction, while healing time was normal in the patients without displaced fractures. Reduced bone turnover may account to delay healing (Keegan et al, 2004).

Prevention and treatment of osteoporosis in diabetic women: At present, few studies specifically addressed prevention and treatment of osteoporosis in diabetic women. In the fracture intervention trial, three years of alendronate therapy resulted in similar improvements in bone density in postmenopausal women with $(n=297)$ or without diabetes. General recommendations regarding the healthy life-style, exercise, calcium and vitamin D supplementation were probably applicable to them (Lunt, 1996).

Diffuse Idiopathic Skeletal Hyperostosis: DISH is a common degenerative enthesopathy affecting both the axial and appendicular skeleton that may occur with the increased frequency in the diabetics. The etiology of DISH is not known. Its possible relationship with diabetes and obesity and its occurrence in patients with acromegaly have led to the evaluation of the pathogenetic role of insulin, insulin-like growth factor, and growth hormone. (Diffuse idiopathic skeletal hyperostosis (DISH) has several synonyms, including ankylosing hyperostosis and Forestier's disease (Utsinger, 1985). It is a noninflammatory disease, with the principal manifestation being calcification and ossification of spinal ligaments and the regions where tendons and ligaments attach to bone (entheses). Diffuse idiopathic skeletal hyperostosis (DISH) is the non-inflammatory 
disease, with the principal manifestation being calcification and ossification of spinal ligaments and peripheral entheses. Radiographic changes characteristic of DISH may occur in the absence of any musculoskeletal symptoms. So, the etiology and pathogenesis of DISH remain obscure. Possible roles for the mechanical factors, dietary components, metabolic disorders, and a genetic predisposition were suggested. The prevalence of DISH varies with the population studied. It is particularly high among the Pima Indians of the United States, is more common in men than women, less common in blacks than Caucasians. The prevalence increased with the age. Pain in the thoracic spine region is a common complaint, being present in 40 to $80 \%$ of patients. Morning stiffness, often considered a symptom of inflammatory back pain, may also be present. Dysphagia sometimes results from impingement of cervical osteophytes upon the pharynx. Ossification of the posterior spinal ligament in the cervical spine may cause spinal cord compression. There are no diagnostic physical findings, but limited motion of the thoracic spine is the most commonly reported abnormality. The distinctive radiographic finding is flowed the linear calcification and ossification along the anterolateral aspects of the vertebral bodies, which continues across the disc space of the thoracic spine. Anatomically these involve the paravertebral ligaments. Calcification or ossification of the posterior longitudinal ligament arises almost solely in the cervical spine and is better visualized with the CT scanning than plain film radiographs. Similar calcification and ossification may be seen at peripheral entheseal sites, including: the shoulder, iliac crest, ischial-tuberosity, trochanters of the hip, tibial tuberosities, patellae, and bones of the hands and/or feet. The clinical diagnosis of DISH is made on the basis of compatible the radiographic findings. The differential diagnosis includes spondylitis deforming (cervical or lumbar spondylitis) and ankylo-sing spondylitis, and these are distinguished by absence of longitudinal spinal ligamentous calcification in the former and the presence of slender, nearly vertical syndesmophytes in the latter. So, radiographs of the thoracic spine as the initial diagnostic study was used. Treatment of DISH is symptomatic and empiric. The following approach was based upon practical experience: Therapeutic modalities (heat, ultrasound, exercises, \& modification of activities of daily living) and orthotics (Grade 2C). Occasional use of acetaminophen for mild pain and of NSAIDs for moderate pain or peripheral joint symptoms of pain and/or stiffness (Grade 2B) was suggested. Painful enthesopathy that is not adequately controlled by oral agents may respond to local injections of glucocorticoids. Surgery is occasionally required for dysphagia due to cervical spurs, cervical myelopathy and/or ossification of posterior longitudinal ligam-ENT, and other rare neurologic manifestations.

\section{Conclusion}

Osteoporosis is a silent disease without any evidence of disease until a fracture occurs. Approximately 200 million people in the world are affected by osteoporosis and 8.9 million fractures occur each year worldwide. It is a progressive disorder defined by loss of bone mass and density that led to an increased risk for the fractures; porous bone.

Osteoporosis often progresses without any symptoms or pain, and the first sign may actually be a fractured bone. Important risk factors for osteoporosis include: 1-Aging. 2Sex women are four times more likely than men to develop osteoporosis, especially the Caucasian and Asian women. 3- Bone structure and body weight. 4- Family history. 5Prior history of broken bones. 6- Smoking. 7- Alcoholism. 8- Certain diseases. 9- Certain medications.

Two types of haematogenous osteomyelitis in elderly are vertebral and long bone osteomyelitis. Osteomyelitis secondary to contiguous foci of infection occur in elders without vascular insufficiency (secondary to pressure ulcers) or with vascular insufficien- 
cy due to diabetes mellitus or peripheral vascular disease from atherosclerosis. Duration of diabetes 1 play a key role given the lower BMD found among patients with diabetes for $>5$ years. In the Iowa, women with type 1 diabetes were 12.25 times more likely to have had a fracture compared to women without diabetes. Gestational diabetes aging and higher oral glucose tolerance test values during pregnancy may be associated with increased bone loss. Type 2 diabetes mellitus is associated with a higher risk of fractures even in normal or increased bone mineral density Women with type 2 diabetes mellitus have a higher risk of fractures despite increased bone mineral density.

\section{Recommendations}

No doubt, the early diagnosis is the key to minimizing or resizing the impact of the osteoporosis on healthcare system. One must focus on identification of high fracture risk among osteoporotic patients, particularly the diabetic ones. The Public Health Authorities must increase national awareness by Broad cast, among school children or by the development of summer campaigns in order to reduce osteoporotic fractures incidence.

To avoid the osteoporosis one must have 1Got enough Vitamin D \& Calcium? 2- Best specific foods to build-up bones. 3- Exercises practicing even walking before sunrise or after sunset to build-up strong bones. 4Measure bone density, and first of all for diabetic patients is a must.

\section{Nursing Role}

The clinically osteoporosis nurse staff specialists could have the unique roles in the prevention and management of osteoporosis. They oversee the patient's plan of care in specialty clinics such as endocrinology or rheumatology. They teach patients, the junior nursing colleagues, other health care workers and the community about osteoporosis and the therapeutic management are the top priority for these nurse specialists. These qualified nurses would establish experts in the major issues related to osteoporosis such as the pathophysiology, the frac- ture risk factors, proper diagnostic tools and their interpretation, treatment and the compliance issues. The education activities include creating patient education materials, by illustrative lectures for professional nursing societies, journal publications and the development of continuing education. Nursing duty to relief: If patient has osteoporosis fracture, treatment must start with focus on decreasing pain. For that modalities like 1ice, 2- heat, 3-ultrasound 4- electric current. She starts with advanced exercises to involve strengthening, balance, weight beating and flexibility exercises.

A question is: could we hope to have an Egyptian clinically osteoporosis nurse?

\section{References}

Ali, AA, Weinstein, RS, Stewart, SA, et al, 2005: Rosiglitazone causes bone loss in mice by suppressing osteoblast differentiation and bone formation. Endocrinology 146:1226-32.

Balci, N, Balci, MK, Tuzuner, S, 1999: Shoulder adhesive capsulitis and shoulder range of motion in type II diabetes mellitus: association with diabetic complications. J. Diabet. Compl. 13:135-9.

Barrett-Connor, E, Holbrook, TL, 1992: Sex differences in osteoporosis in older adults with non-insulin dependent diabetes mellitus. JAMA 268:3333-40.

Basu, A, Jensen, MD, McCann, F, et al, 2006: Effects of pioglitazone versus glipizide on body fat distribution, body water content, and hemodynamics in type 2 diabetes. Diabetes Care 29: 510-15.

Baumhauer, JF, O'Keefe, RJ, Schon, L, Pinzur, M, 2006: Cytokine-induced osteoclastic bone resorption in charcot arthropathy: An immunohistochemical study. Foot Ankle Int. 27:797-9.

Bhavsar, S, Nimigan, A, Hackam, DG, O'Gorman, DB, Gan, B, et al, 2009: Keloid scaring, but not Dupuytren's contracture, is associated with unexplained carotid atherosclerosis. Clin. Invest. Med. 32, 2:E95-102.

Bogacka, I, Xie, H, Bray, G, Smith, S, 2004: Effect of pioglitazone on peroxisome proliferator-activated receptor-gamma target genes related to lipid storage in vivo. Diabetes Care 27: 1660-8.

Cagliero, E, Apruzzese, W, Perlmutter, GS, Nathan, DM, 2002: Musculoskeletal disorders 
of the hand and shoulder in patients with diabetes mellitus. Am. J. Med. 112:487-92.

Compston, JE, Smith, EM, Matthews, C, Schofield, P, 1994: Whole body composition and regional bone mass in women with insulin- dependent diabetes mellitus. Clin. Endocrinol. (OXF) 41:289-92.

Dominguez, ,LJ, Muratore, M, Quarta, E, Zagone, G, Barbagallo, M, 2004: Osteoporosis and diabetes. Reumatismo. 56, 4:235-41.

El-Tawdy, AHF, Ibrahim, EA, Abdallah, ES, Saleh, AMA, Al-Sakhawy, EMA, Morsy, TA, 2015: General evaluation of the diabetic foot. Egyptian Military Medical Journal 70, 2:51-67.

Fisher, L, Kurtz, A, Shipley, M, 1986: Association between cheiroarthropathy and frozen shoulder in patients with insulin dependent diabetes mellitus. Br. J. Rheumatol. 25:141-8.

Forst, T, Pfutzner, A, Kann, P, et al, 1995: Peripheral osteopenia in adult patients with insulin-dependent diabetes mellitus. Diabet. Med. 12:871-8.

Gamstedt, A, Holm-Glad, J, Ohlson, CG, Sundstrom, M, 1993: Hand abnormalities are strongly associated with the duration of diabetes mellitus. J. Int. Med. 234:189-92.

Graham, RG, Hudson, DA, Solomons, M, Singer, M, 2004: A prospective study to assess the outcome of steroid injections and wrist splinting for the treatment of carpal tunnel syndrome. Plast. Reconstr. Surg.113:550-9.

Gruson, LM, Franks, A Jr, 2005: Scleredema and diabetic sclerodactyly. Dermatol. Online J. 11, 4:3-9.

Guan, Y, Hao, C, Cha, DR, et al, 2005: Thiazolidinediones expand body fluid volume through PPARgamma stimulation of ENaC-mediated renal salt absorption. Nat Med 11:861-9.

Gulliford, MC, Latinovic, R, Charlton, J, Hughes, RA, 2006: Increased incidence of carpal tunnel syndrome up to 10 years before diagnosis of diabetes. Diabetes Care 29:1929-32 Chaudhuri, KR, Davidson, AR, Morris, IM, 1989: Limited joint mobility and carpal tunnel syndrome in insulin-dependent diabetes. Br. J. Rheumatol. 28:191-8.

Hernigou, P, Guissou, I, Homma, Y, Poignard A, Chevallier, N, et al, 2015: Percutaneous injection of bone marrow mesenchymal stem cells for ankle non-unions decreases complications in patients with diabetes. Int. Orthop. 39, 8:163943.

Hochberg, MC, 1991: Epidemiology of osteo- arthritis: Current concepts and new insights. J. Rheumatol. 18, 27:S4-9.

Home, PD, Pocock, SJ, Beck-Nielsen, H, et al, 2009: Rosiglitazone evaluated for cardiovascular outcomes in oral agent combination therapy for type 2 diabetes (Record): A multicentre, randomized, open-label trial. Lancet 373. 9681: 2125-35.

Horn, CA, Bradley, J, Brandt, K, et al, 1992: Impairment of osteophyte formation in hyperglycaemic patients with Type II diabetes mellitus and knee osteoarthritis. Arthritis Rheum. 35: 336-9.

Janghorbani, M, Van Dam, RM, Willett, WC, Hu, FB, 2007: Systematic review of type 1 and type 2 diabetes mellitus and risk of fracture. Am. J. Epidemiol.166:495-9.

Jara, A, Bover, J, Felsenfeld, AJ, 1995: Development of secondary hyperparathyroidism and bone disease in diabetic rats with renal failure. Kidney Int.47:1746-9.

Jelinek, JE, 1993: The skin in diabetes. Diabet. Med. 10:201-8.

Kapoor, A, Sibbitt, WL Jr, 1989: Contractures in diabetes mellitus: Syndrome of limited joint mobility. Semin. Arthritis Rheum. 18:168-72.

Kayath, MJ, Dib, SA, Vieiaa, J, 1995: Prevalence and magnitude of osteopenia associated with insulin-dependent diabetes mellitus. J. Diabetes Complicat. 1994; 8:97-9.

Keegan, TH, Schwartz, AV, Bauer, DC, et al, 2004: Effect of alendronate on bone mineral density and biochemical markers of bone turnover in type 2 diabetic women: the fracture intervention trial. Diabetes Care 27:1547.

Kendall, DM, Rubin, CJ, Mohideen, P, et al, 2006: Improvement of glycemic control, triglycerides, and HDL cholesterol levels with muraglitazar, a dual (alpha/gamma) peroxisome proliferator-activated receptor activator in patients with type 2 diabetes inadequately controlled with metformin monotherapy: A double-blind, randomized, pioglitazone-comparative study. Diabet. Care 29:1016-21.

Khan, TS, Fraser, LA, 2015: Type 1 diabetes and osteoporosis: from molecular pathways to bone phenotype. J. Osteoporos 2015:174186. doi: 10.1155/2015/174186.

King, A, Armstrong, D, Chinnapongse, S, 2003: Clinical observations of weight gain associated with pioglitazone: 3 years. Diabetes 52, 1:S123-8.

Kozin, F, 1998: Painful shoulder and the reflex 
sympathetic dystrophy syndrome. In: Arthritis and Allied Conditions, $11^{\text {th }}$ edition, McCarty, DJ (Ed), Lea \& Febiger, Philadelphia.

Krakauer, JC, Malachi, J, McKenna, NFB, et al, 1995: Bone loss and bone turnover in diabetes. Diabetes 44:775-80.

Lecka-Czernik, B, Ackert-Bicknell, C, Adamo, M, et al, 2007: Activation of peroxisome proliferator-activated receptor gamma (PPARgamma) by rosiglitazone suppresses components of insulin-like growth factor regulatory system in vitro and in vivo. Endocrinology 148: 903-9.

Leslie, WD, Lix, LM, Prior, HJ, et al, 2007: Biphasic fracture risk in diabetes: a populationbased study. Bone 40:1595-9.

Lettgen, B, Hauffa, B, Mohlmann, C, et al, 1995: Bone mineral density in children and adolescents with juvenile diabetes: Selective measurement of bone mineral density of trabecular and cortical bone using peripheral quantitative computed tomography. Horm. Res. 43:173-80.

Loder, RT, 1988: The influence of diabetes mellitus on the healing of closed fractures. Clin. Orthop. 232: 211-8.

Lunt, H, 1996: Women and diabetes. Diabet. Med.13:1009-14.

Mastrandrea, LD, Wactawski, WJ, Donahue, RP, et al, 2008: Young women with type 1 diabetes have lower bone mineral density that persists over time. Diabetes Care 31:1729-34.

Mavrikakis, ME, Sfikakis, PI, Kontoyannis, S A, et al, 1999: Clinical and laboratory parameters in adult diabetics with and without calcific shoulder periarthritis. Calcif. Tiss. Int. 49:288-92.

McNair, P, 1988: Bone mineral metabolism in human type 1 (insulin dependent) diabetes mellitus. Dan. Med. Bull. 35:109-14.

Mimata, Y, Sato, K, Tokunaga, K, Tsukimura, I, Tada, H, et al, 2015: Diabetic muscle infraction of the tibialis anterior and extensor hallu cis longus muscles mimicking the malignant soft-tissue tumor. Case Rep. Orthop. 2015: 656307. doi: 10.1155/2015/656307

Moren-Hybbinette, I, Moritz, U, Schersten, B, 1987: The clinical picture of the painful diabetic shoulder, natural history, social consequences and analysis of concomitant hand syndrome.

Acta Med. Scand. 221:73-8.

Nesto, RW, Bell, D, Bonow, RO, et al, 2003: Thiazolidinedione use, fluid retention, and congestive heart failure: a consensus statement from the American Heart Association and American Diabetes Association. Circulation 108:2941-9
Noble, J, Heathcote, JG, Cohen, H, 1984: Diabetes mellitus in the aetiology of Dupuytren's disease. Br. J. Bone Joint Surg. 66:322-8.

Olmos, JM, Perez-Castrillon, JL, Garcia, M T, et al, 1994: Bone densitometry and biochemical bone remodelling markers in type 1 diabetes mellitus. Bone Miner; 26:1-8.

Pal, B, Anderson, J, Dick, WC, Griffiths, ID, 1986: Limitation of joint mobility and shoulder capsulitis in insulin and non-insulin dependent diabetes mellitus. Br. J. Rheumatol. 25:147-52.

Pei, Y, Hercz, G, Greenwood, C, et al, 1993: Renal osteodystrophy in diabetic patients. Kidney Int. 44:159-62.

Pierre-Jerome, C, Reyes, E, Moncayo, V, Chen, ZN, Terk, MR, 2012: MRI of cuboid bone: analysis of changes in diabetic versus non-diabetic patients and their clinical significance. Eur. J. Radiol. 81, 10:2771-5.

Redmond, CL, Bain, GI, Laslett, LL, McNeil, JD, 2012: Deteriorating tactile sensation in patients with hand syndromes associated with diabetes: a two-year observational study. J. Diabetes Complicat. 26, 4:313-8.

Rishaug, U, Birkeland, K, Falch, J, Vaaler, S, 1995: Bone mass in non-insulin dependent diabetes mellitus. Scand. J. Clin. Lab. Invest. 55:257.

Rzonca, SO, Suva, LJ, Gaddy, D, et al, 2004: Bone is a target for the antidiabetic compound rosiglitazone. Endocrinology 145:401-9.

Seibold, J, 1982: Digital sclerosis in children with insulin dependent diabetes mellitus. Arthritis Rheum. 25:1357-62.

Selby, PL, Shearing, PA, Marshall, SM, et al, 1995: Hydroxyproline excretion is increased in diabetes mellitus and related to the presence of microalbuminuria. Diabet Med 12:240-9.

Shadid, S, Jensen, MD, 2003: Effects of pioglitazone versus diet and exercise on metabolic health and fat distribution in upper body obesity. Diabetes Care 26:3148-52.

Sibbitt, WL, 1989: Fibrosing syndromes: diabetic stiff hand syndrome, Dupuytren's contracture and plantar fasciitis. In: Arthritis and Allied Conditions, $11^{\text {th }}$ ed., McCarty, DJ (Ed), Lea \& Febiger, Philadelphia.

Singleton, JR, Smith, AG, Marcus, RL, 2015: Exercise as therapy for diabetic and pre-diabetic neuropathy. Curr. Diab. Rep. 15, 12:120-6.

Strotmeyer, ES, Cauley, JA, Schwartz, AV, et $\boldsymbol{a l}, 2004$ : Diabetes is associated independently of body composition with BMD and bone volume in older white and black men and women: The 
health, aging, and body composition study. J. Bone Miner Res. 19:1084-90.

Sun, F, Ito, M, et al, 2000: Efficacy of troglitazone on body fat distribution in type 2 diabetes. Diabetes Care 23:1067.

Tuominen, JT, Impivaara, O, Puukka, P, Ronnemaa, T, 1999: Bone mineral density in the patients with type 1 and type 2 diabetes. Diabetes Care 22:1196-204.

Utsinger, PD, 1985: Diffuse idiopathic skeletal hyperostosis. Clin/ Rheum. Dis.11:325-9.

Van Daele, PLA, Stolk, RP, Burger, H, et al, 1996 Bone density in non-insulin dependent diabetes mellitus. Ann. Intern. Med. 122:409-14.

Vestergaard, P, Rejnmark, L, Mosekilde, L, 2005: Relative fracture risk in patients with diabetes mellitus, and the impact of insulin and oral antidiabetic medication on relative fracture risk.
Diabetologia 48:1292-9.

Wakasugi, M, Wakao, R, Tawata, M, et al, 1993: Bone mineral density measured by dual energy X-ray absorptiometry in patients with non-insulin dependent diabetes mellitus. Bone 14:29-34.

Yamamoto, M, Yamaguchi, T, Yamauchi, M, et al, 2009: Diabetic patients have an increased risk of vertebral fractures independent of BMD or diabetic complications. J. Bone Miner. Res. 24:702-8.

Yki-Jarvinen, H, 2004: Drug Therapy: Thiazolidinediones. N. Engl. J. Med. 351:1106-12.

Yosipovitch, G, Yosipovitch, Z, Karp, M, Mukamel, M, 1990: Trigger finger in young patients with insulin dependent diabetes. J. Rheumatol.17:951-60.
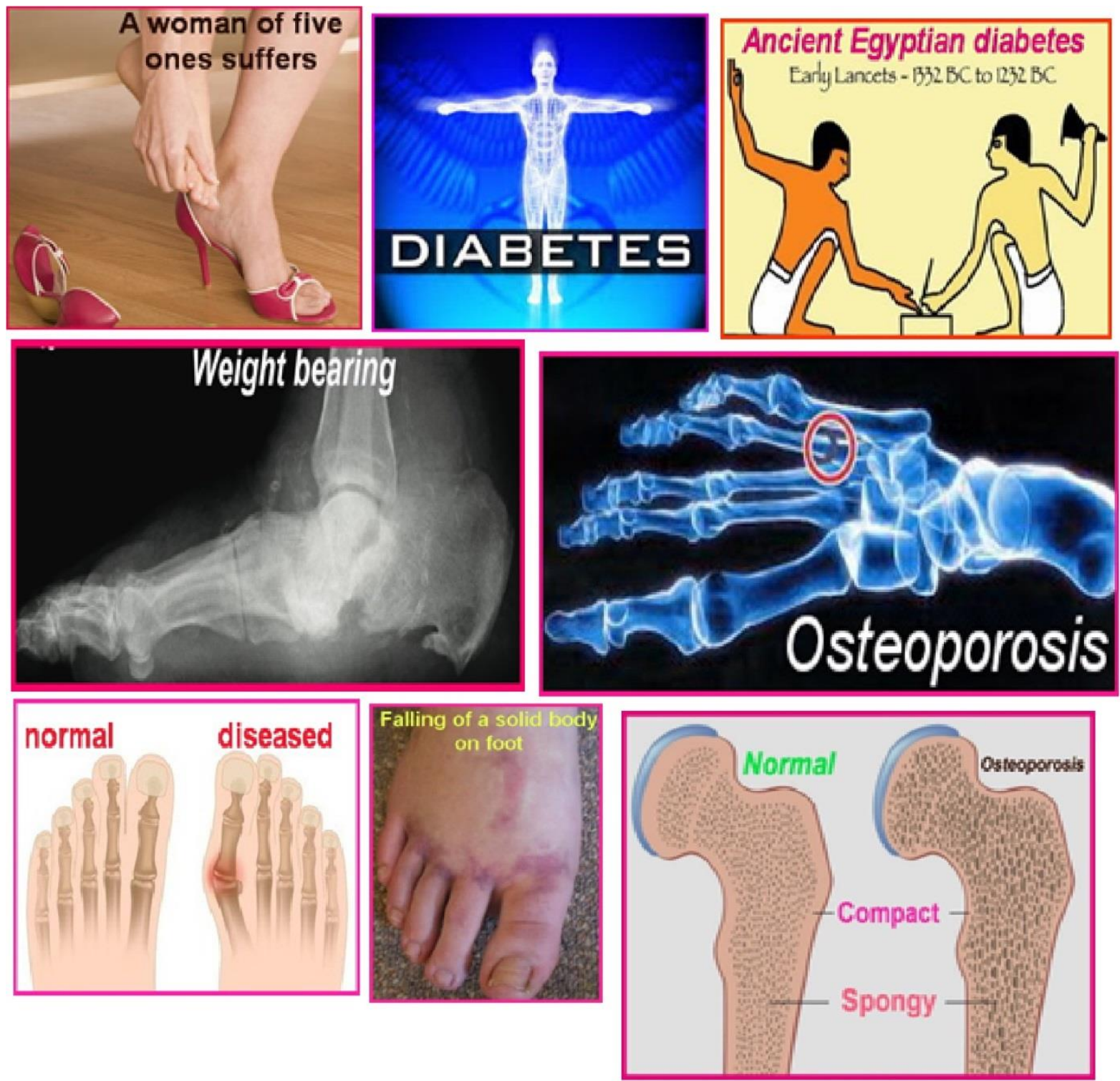